\title{
Ghrelin, Diabetes and Obesity - Prospects for an Anti-obesity Vaccine
}

The prevalence of diabetes is increasing worldwide and nowadays stands as a major public health problem. Undoubtedly, the 21st century has the most diabetogenic environment in human history. ${ }^{1}$ The number of people living with diabetes has soared to 366 million, and the disease kills one person every seven seconds, posing a "massive challenge" to healthcare systems worldwide. ${ }^{2}$ Diabetes is a huge and growing problem, and the costs to society are high and escalating, affecting all countries. Over the past 25 years or so, the prevalence of type 2 diabetes in the USA has almost doubled, with three- to five-fold increases in India, Indonesia, China, Korea and Thailand. ${ }^{3}$

Propelling the upsurge in cases of diabetes (and hypertension) is the growing prevalence of overweight and obesity which is emerging threats to the health of both developed and developing world. ${ }^{4}$ Apart from type 2 diabetes, obesity endangers the lives of millions of people worldwide, through other comorbidities like, heart disease, cancers stroke, arthritis, and major depression. Obesity is a complex disease, caused by the interplay between environmental and genetic factors.

Research over the last one and a half decade has revolutionized understanding of the molecular mechanisms that control body weight and fat. The regulation of body weight is homeostatic. On an individual, long-term basis, energy balance is remarkably precise, particularly when considering the large day-to-day variation in food intake and energy expenditure. The hypothalamus is the key region in the central nervous system involved in appetite regulation, though other brain regions also play a role. The increase in hunger before a meal and the reduction following it (postprandial satiety) might be explained by a gut sensor system, signalling from the gut to the appetite centres in the brain. Both neural and endocrine afferent signalling appear to be involved.

The gastrointestinal tract is the largest endocrine organ in the body and an important source of regulatory peptide hormones. ${ }^{5}$ The gut peptide secretin was the first substance given the name "hormone." Early studies into the gut endocrine system focused on the role of gut hormones in the peripheral regulation of gastrointestinal function, for example, secretin on pancreatic secretion, cholecystokinin on gall bladder contraction, and gastrin on gastric acid release. It was not until the 1970s, a period during which a number of novel gut hormones were identified, that it became clear that gut hormones signalled to the central nervous system (CNS), often in profound and subtle ways. In 1973, cholecystokinin became the first gut hormone demonstrated to influence appetite, paving the way for many seminal studies into the role of the brain-gut axis in energy homeostasis. $^{6}$

Ghrelin is one of the circulating enteric peptides, produced by the X/A-cells of oxyntic glands, abundantly present in the mucosal layer of the fundus region of the stomach. Interestingly the receptor for this hormone was known before the actual ligand was discovered. The discovery of ghrelin began three decades ago with the identification of several synthetic compounds which could release growth hormone from the pituitary somatotrophs by stimulating the GH secretagogue receptor (GHS) till it was finally identified from rat stomach in 1999 as 28-aminoacid peptide. ${ }^{7-9}$ The human ghrelin gene is localized on chromosome 3p25-26. Ghrelin circulates in body in active (acylated) and inactive (desacylated) forms. The physiological role of desacylated ghrelin is not clear. The des-acyl ghrelin can neither bind GHS-R nor exhibit GH-releasing activity in rats.

Ghrelin is an orexigenic hormone which stimulates appetite and regulates energy balance, and thus is one of the candidate genes for obesity and T2DM. Being the only known circulating appetite stimulant, ghrelin has garnered widespread attention, as reflected by more than 5000 scientific papers featuring ghrelin that have been published since the first reports of its orexigenic actions. During the last years both basic research and genetic association studies have revealed association between the ghrelin gene and obesity, metabolic syndrome or type 2 diabetes. ${ }^{10}$ In this issue of the Journal of Medical Sciences also, there is a study by Misra et al, wherein the authors have estimated plasma active ghrelin levels in patients with type 2 diabetes in an attempt to establish the relationship of ghrelin with insulin resistance, hypertension, dyslipidemia and obesity. The authors found a significant negative association of fasting plasma active ghrelin levels with fasting insulin levels, insulin resistance, diabetes mellitus and obesity (BMI $>25)$.

Plasma ghrelin levels exhibits a pronounced diurnal variation and are increased by fasting and before meals and at night, while food intake causes rapid suppression especially high-calorie or high-carbohydrate meals. ${ }^{11}$ The exact mechanisms that mediate suppression of systemic ghrelin secretion by food are not known but blood glucose level may be the most probable candidate because oral or intravenous administration of glucose decreases plasma ghrelin concentration. ${ }^{12}$ Ghrelin is 
only a hunger signal from peripheral tissues. Intravenous and subcutaneous injections of ghrelin increase food intake. Ghrelin infusion that leads to an increase in plasma ghrelin to preprandial levels stimulates hunger and spontaneous food intake. ${ }^{13}$ However the postprandial suppression normally expected does not happen in obese individuals thereby contributing to obesity. Long-term ghrelin administration has been shown to cause weight gain stimulating food intake, spontaneous activity, and promoting adipogenesis. Variations in polymorphism for ghrelin gene has been shown to affect human adiposogenesis.

Diet and exercise are still the keystone for treatment of obesity and clinicians have few medical therapeutic means to fight obesity. Current anti-obesity drugs seldom achieve significant weight loss that is sustainable. Often there is weight regain as most drugs are prone to tolerance or have side effects that limit their chronic use. ${ }^{14}$ Bariatric surgery is the only treatment with demonstrated efficacy, providing greater and more sustainable weight reduction than behavioural and pharmacological interventions, but is only indicated in individuals with a BMI of over 40 or of 35 with comorbidities. ${ }^{14}$ Therefore new medical treatment approaches to control body weight remain a high priority.

In the battle to treat the pandemic of obesity, one therapeutic strategy is to block endogenous signals that stimulate appetite and control body weight. Since ghrelin is the only orexigenic hormone identified so far, it has been a promising target in the development of new treatments for obesity. The relative importance of ghrelin in long-term body-weight regulation is uncertain, however, because genetic and pharmacologic blockade of ghrelin signalling have yielded variable results to date. Using a novel approach of vaccinating rats against their own ghrelin, it was found that animals with high ghrelin-specific antibody titers displayed restricted body weight, without evidence of non-specific inflammation following the vaccine. ${ }^{15}$ Anti-ghrelin vaccines using bovine serum albumin as immunogenic substances also decreased bodyweight gain by decreasing food intake and bodyweight in pigs. ${ }^{16}$ However, these vaccines required the use of immune adjuvants with restricted human utilization.

Finally, counteracting ghrelin through an anti-ghrelin vaccine might prove to be a useful tool for obesity treatment to be used in association with diet and exercise, but is unlikely to be an alternative to dieting. A question waiting to be answered is for how long the immunoneutralization would be efficacious. In addition, the long-term effects of prolonged ghrelin neutralization need to be considered as well as whether there will be risk for the vaccine to interfere with other physiological actions of ghrelin.

Shariq Rashid Masoodi, MD, DM

(Executive Editor)

\section{References}

1. Zimmet P, K Alberti and J Shaw. Global and societal implications of the diabetes epidemic. Nature 2001;414: 782-7.
2. World Diabetes Atlas 5th edn. 2011 International Diabetes Federation, Brussels. http://www.idf.org/ diabetesatlas/news/fifth-edition-release (Accessed November 2011).

3. Yoon K, et al. Epidemic obesity and type 2 diabetes in Asia. Lancet 2006; 368:1681-8.

4. Hossain P, et al. Obesity and Diabetes in the Developing World - A Growing Challenge. New Engl J Med 2007;356 (3):213.

5. Murphy KG, Dhillo WS, Bloom SR. Gut Peptides in the Regulation of Food Intake and Energy Homeostasis. Endocrine Reviews 2006;27(7):719-727.

6. Gibbs J, Young RC, Smith GP. Cholecystokinin decreases food intake in rats. J Comp Physiol Psychol 1973; 84:488-495.

7. Kojima M, Hosoda H, Date Y, Nakazato M Matsuo, H and Kangawa K. Ghrelin is a growth-hormone-releasing acylated peptide from stomach. Nature 1999;402:656-60.

8. Civelli O. Functional genomics: the search for novel neurotransmitters and neuropeptides. FEBS Lett 1998; 430:55-58.

9. Kojima M, Hosoda H, Matsuo H, Kangawa K. Ghrelin: discovery of the natural endogenous ligand for the growth hormone secretagogue receptor. Trends Endocrinol Metab 2001;12:118-22.

10. Pulkkinen L, Ukkola O, Kolehmainen M, Uusitupal M. Ghrelin in Diabetes and Metabolic Syndrome. Int J Pept 2010;2010. pii: 248948. Epub2010 Apr 27.

11. Tschop M, Wawarta R, Riepl RL, et al. Post-prandial decrease of circulating human ghrelin levels.J Endocrinol Invest 2001;24:RC19-RC21.

12. Shiiya T, Nakazato M, Mizuta M, Date Y, Mondal MS, Tanaka M, Nozoe S, Hosoda H, Kangawa K and Matsukura S. Plasma ghrelin levels in lean and obese humans and the effect of glucose on ghrelin secretion. $J$ Clin Endocrinol Metab 2002; 87, 240-44.

13. Wren AM, Seal LJ, Cohen MA, et al. Ghrelin enhances appetite and increases food intake in humans. J Clin Endocrinol Metab 2001;86:5992.

14. Snow V, Barry P, Fitterman N, Qaseem A, Weiss K. Pharmacologic and surgical management of obesity in primary care: a clinical practice guideline from the American College of Physicians. Ann Intern Med 2005; 142(7): 525-531.

15. Zorrilla EP, Iwasaki S, Moss JA et al. Vaccination against weight gain. Proc Natl Acad Sci USA 2006;103(35): 13226-31.

16. Vizcarra JA, Kirby JD, Kim SK, Galyean ML. Active immunization against ghrelin decreases weight gain and alters plasma concentrations of growth hormone in growing pigs. Domest Anim Endocrinol 2007;33(2):176-89. 